TITLE:

\title{
Automated Violin Fingering Transcription Through Analysis of an Audio Recording
}

\section{$\operatorname{AUTHOR}(S):$}

Maezawa, Akira; Itoyama, Katsutoshi; Komatani, Kazunori; Ogata, Tetsuya; Okuno, Hiroshi G.

\section{CITATION:}

Maezawa, Akira ...[et al]. Automated Violin Fingering Transcription

Through Analysis of an Audio Recording. Computer Music Journal 2012, 36(3): 57-72

ISSUE DATE:

2012-08-14

URL:

http://hdl.handle.net/2433/198554

RIGHT:

(C) 2012 Massachusetts Institute of Technology. 


\section{Akira Maezawa, Katsutoshi Itoyama, Kazunori Komatani, Tetsuya Ogata, and Hiroshi G. Okuno}

Department of Intelligence Science

and Technology

Kyoto University Graduate School of Informatics

Yoshida Honmachi

Sakyo, Kyoto 606-8501, Japan

akira_maezawa@gmx.yamaha.com

itoyama@kuis.kyoto-u.ac.jp

komatani@nuee.nagoya-u.ac.jp

\{ogata, okuno\}@i.kyoto-u.ac.jp

\section{Automated Violin Fingering Transcription Through Analysis of an Audio Recording}

\begin{abstract}
We present a method to recuperate fingerings for a given piece of violin music in order to recreate the timbre of a given audio recording of the piece. This is achieved by first analyzing an audio signal to determine the most likely sequence of two-dimensional fingerboard locations (string number and location along the string), which recovers elements of violin fingering relevant to timbre. This sequence is then used as a constraint for finding an ergonomic sequence of finger placements that satisfies both the sequence of notated pitch and the given fingerboard-location sequence.

Fingerboard-location-sequence estimation is based on estimation of a hidden Markov model, each state of which represents a particular fingerboard location and emits a Gaussian mixture model of the relative strengths of harmonics. The relative strengths of harmonics are estimated from a polyphonic mixture using score-informed source segregation, and compensates for discrepancies between observed data and training data through mean normalization.

Fingering estimation is based on the modeling of a cost function for a sequence of finger placements. We tailor our model to incorporate the playing practices of the violin.

We evaluate the performance of the fingerboard-location estimator with a polyphonic mixture, and with recordings of a violin whose timbral characteristics differ significantly from that of the training data. We subjectively evaluate the fingering estimator and validate the effectiveness of tailoring the fingering model towards the violin.
\end{abstract}

In musical instrument performance, deciding the sequence of finger placements needed to produce a given sequence of pitches, known as the fingering, is an important and sometimes difficult problem that musicians need to solve. Fingering decisions can be difficult because the fingering must be both musical and ergonomic, two often-conflicting ideals. For example, the "easiest" fingering on a violin often involves unmusically abrupt changes of timbre. This is because most pitches can be found in more than one location on the instrument (i.e., on more than one string). Each string, however, has a different timbre (for reasons we explain later), and often the easiest fingering involves changes between strings. An experienced musician would choose a balanced fingering that not only satisfies ergonomic finger placements but also expresses the musician's artistic values.

The essence of violin fingering resides in finding both an ergonomic sequence of finger placements

Computer Music Journal, 36:3, pp. 57-72, Fall 2012

(c) 2012 Massachusetts Institute of Technology. and a musically appropriate fingerboard location sequence, the sequence of locations on the fingerboard on which the finger presses the string. Each fingerboard location specifies both the longitudinal location along the string and the latitudinal position, i.e., which of the four strings is played. Each string is tuned differently and has a distinct timbre. Therefore, it is essential for a violinist to choose a fingerboard-location sequence that sounds musically well-motivated. For example, in Air on the $G$ String, August Wilhelmi's well-known arrangement of the second movement of J. S. Bach's Orchestral Suite No. 3, the arranger specifies the entire solo violin part to be played on one string (the G string), most likely to maintain the consistent, warm timbre that is characteristic of the G string.

This study aims to develop a method for analyzing an audio recording of a violin and estimating the fingering required to recreate the "sound" of a particular artist's performance. Such a method would allow a beginner, for example, to analyze the recordings of past masters and gain insights on how to imitate them. It could also help violin students 
to appreciate different musical values by comparing fingerings played by different musicians. Finding similarities of fingering among musicians would allow one to find a stylistically suitable way to play a particular piece, a difficult task for a violinist studying alone without a teacher's help.

Our goal requires (1) analyzing an audio signal to estimate the fingerboard location sequence, and (2) choosing an ergonomic finger placement sequence that satisfies the sequence of notated notes and the estimated fingerboard location. Existing methods in fingering estimation do not focus on timbral differences caused by different fingerboard locations, however, and existing methods for estimating the fingerboard-location sequence need robustness in a polyphonic mixture.

Most studies in fingering-estimation research intend not to retrieve a fingering from a particular recording, but rather to determine an ergonomic fingering. In these studies, the general framework involves designing a cost function for moving the hand from one shape to another, and finding a sequence of hand movements that minimizes the accrued cost. In the seminal work by Sayegh (1989), the cost uses the distance that the hand traverses horizontally and vertically across the instrument. Other work involves similar kinds of costs based on the actual distance that the hand needs to traverse based on different constraints posed by different instruments, such as the guitar (Radicioni, Anselma, and Lombardo 2004) or the piano (Kasimi, Nichols, and Raphael 2007; Yonebayashi, Kameoka, and Sagayama 2007). The costs may be given a priori or learned using training data (Radisavljevic and Driessen 2004). The formulation of fingering estimation as a cost-minimization problem has been given a probabilistic interpretation using hidden Markov models (HMM; Yonebayashi, Kameoka, and Sagayama 2007). These studies all seem to have the perspective that musicality resides in the sequence of notated notes, which overlooks the expression added by the performer. Such an orientation implies that given a symbolic representation of music, a fingering may be determined on a one-to-one basis. Each person, however, given a piece of music, might play it using a different fingering based on his or her musical values and physiological constraints. Clearly, performance analysis based on an audio signal or video is essential for realizing our goal of reconstructing fingerings from recorded performances.

Studies in detailed performance analysis fail when the music in the audio recording is polyphonic, as well as when the sound of the instrument that needs to be analyzed is significantly different from the training data. In practical situations, however, it is essential to be able to analyze a musical phrase within a polyphonic mixture. Methods for analyzing audio spectra to determine the control input for the violin (Krishnaswamy and Smith 2003; Barbancho 2009) or the guitar (Traube and Smith 2000) do not work well on instruments with acoustic characteristics other than those used in training, or make highly restrictive assumptions on how the instrument is played. Greater accuracy is reported using audiovisual fusion (Zhang, Zhu, and Leow 2007; Lu et al. 2008). Some studies use only visual information to analyze the fingering of a guitar (Burns and Wanderley 2006). In either case, the necessity of video severely limits the kind of musical recordings that can be analyzed.

In this article, we develop a method to recuperate the fingering from an audio recording of a violin in a polyphonic mixture by analyzing the audio recording and finding an ergonomic fingering that captures the particular recording's timbre as expressed in a specific fingerboard-location sequence. Our method is a two-step procedure. First, it analyzes the audio signal to estimate the fingerboard location sequence. Second, it determines an ergonomic fingering that satisfies the fingerboard-location sequence. When analyzing the fingerboard-location sequence, we use features that are robust in a polyphonic mixture. Moreover, the method uses a feature-adaptation mechanism to improve the robustness when the instrument sounds are different from the ones used for training. For the ergonomic fingering decision, we incorporate a cost function that reflects practices of violin playing (which is not necessarily applicable to other stringed instruments such as the guitar or the cello). We evaluate the performance of the fingerboard-location estimator and the fingeringdecision method. 


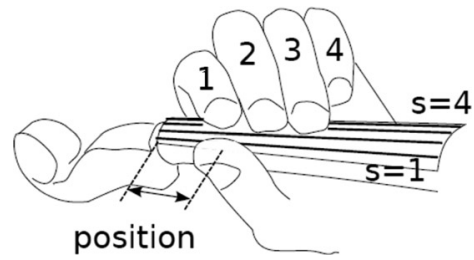

\section{Violin Fingering: A Primer}

We shall briefly review the fundamentals of violin fingering and its terminology, as these play important roles in understanding our method. The left hand defines the pitch and the string on which a note is played. Although there are two defining factors in fingering (i.e., finger and string), violinists often talk about fingering also in terms of the left hand position, i.e., the general placement of the left hand required to play a given note on a certain string using a certain finger. As shown in Figure 1, in this article we associate the string on which a note is played with the variable $s$, where $s=1$ is the lowest-tuned string and $s=4$ is the highest. These strings are conventionally tuned seven semitones apart, where $s=3$ is typically tuned to A $4=440 \mathrm{~Hz}$. The strings are typically notated in a music score using a Roman numeral, where the lowest string $(s=1)$ is associated with IV, and the highest $(s=$ 4) with I. Placed fingers are labeled by numbers, where 1 refers to the index finger, 2 the middle, 3 the ring, and 4 the little finger. An open string (i.e., no finger is pressed and the string vibrates at the tuned pitch) is referred to by 0 ; only four pitches on a violin can be played as an open string. In this article, the position is defined by the number of semitones that the first finger must traverse from the nut (the ridge over which the string passes on the end of the fingerboard near the tuning pegs) in order to play a particular fingering.

Finger placement is determined by considering technical ease and musical effect. Using a certain finger (e.g., the index finger instead of the little finger) facilitates execution of some musical effects related to pitch, such as a smooth transition between two notes (glissando), or a low-frequency modulation of a note (vibrato). At the same time, some sequences of finger placement are easier to execute than others. For example, rapid movement of the little finger is considerably more tiresome than that of the index finger. The choice of the string on which to play a given note is determined by considering the consistency of sonority. For example, because each string has a distinct sonority, violinists often play on one string to prevent abrupt changes of the sound quality. The difference in sonority when a given pitch is played on one string versus another results from (1) the differences in the physical attributes of each string itself, such as the diameter, tension, and material; (2) differences in the position along the string at which the finger must be placed (because each string is tuned differently); and (3) in cases when the pitch is available as an open string, the difference between the rigid termination provided by the nut and the soft termination provided by the finger.

The choice of string is the one factor in fingering that is most likely to produce audible differences. By contrast, the position is sometimes chosen for visual effect and to demonstrate the violinist's technical skills. For example, the violinist may present a "flashy" playing style through a wide change of position.

The consistency of sonority offered by playing on one string often forms a trade-off with the consistency of position that facilitates playing. In a fast piece, abrupt changes of sonority caused by a certain fingering may be overlooked if it simplifies playing. On the other hand, in a slow piece, a violinist may choose a difficult fingering that produces a certain sonority. A violinist may, for example, value consistency of sonority in a slow, "singing" (cantilena) passage by playing on one string.

\section{Method}

Our method is based on determining the fingerboardlocation sequence from a polyphonic audio mixture that contains a musical phrase for solo violin, and finding a sensible fingering that satisfies the estimated fingerboard-location sequence. We assume the violin plays a monophonic melody using normal bowing technique (i.e., we do not consider extended 
Figure 2. System block

diagram.

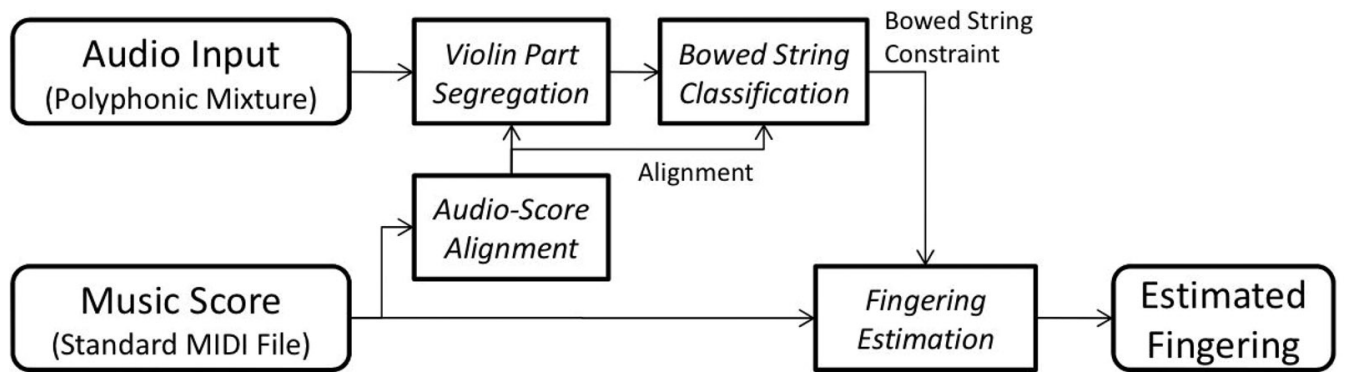

playing technique such as plucking [pizzicato], striking the string with the wooden side of the bow [col legno],or playing very close to the bridge to produce a shimmering sound [sul ponticello]).

Our task involves three aspects:

1. Designing a feature that performs well in a polyphonic mixture.

2. Designing a scheme such that the performance does not degrade from an acoustical mismatch with the training data, whether because of characteristics of the violins, the rooms, or the recording process.

3. Designing a fingering model that reflects the practices of violin performance.

We attack the first problem by fitting a sum-ofsinusoids plus noise model to the observed spectrum and using the estimated harmonic parameters as the feature. The second problem is attacked by normalizing the average features of the training set and by doing the same for the recording whose fingerboard-location sequence we would like to identify. We solve the third problem by introducing a new model of violin fingering, the violin pedagogical model, which incorporates features that are inspired by practices of violin playing. The system-level block diagram is shown in Figure 2.

\section{Fingerboard-Location Sequence Estimation through Viterbi Alignment}

In order to estimate a sensible fingering from the audio, the fingerboard-location sequence must be estimated from a polyphonic mixture of sound that contains a violin melody. Because the string on which a note is played (the bowed string) is the primary factor that influences the timbre for different fingerboard locations, we would like to estimate the sequence of bowed strings. Our estimation method is based on a bowed-string classifier using features that are robust to polyphonic accompaniment, and a classifier that takes into account the playability of a particular fingerboardlocation sequence.

\section{Feature Extraction by Harmonic-Model Fitting}

We extract, from a polyphonic audio mixture that contains a violin melody, the relative strengths of the first $N$ violin harmonics (partials), where $N=$ 10 , the first partial is the fundamental frequency, and the others are overtones. The parameter is dependent on the material property of the string, the body resonance characteristics, and on how the instrument is played (e.g., bow force, bow velocity, and the contact point of the bow and the string [Cremer and Allen 1984; Fletcher and Rossing 1998]). The feature extraction involves fitting a sum of harmonically spaced sinusoids onto the observed short-time Fourier transform representation of the input audio signal, $Y(f, t)$, where $f$ is the frequency bin and $t$ is the time index. Because the input contains not only the harmonic sound of the violin but also transients of the violin and accompaniments, the harmonic sound of the violin part must be segregated. This is achieved by generating a time-frequency mask that passes the harmonic component of the violin part, based on the observed spectrogram, the music score given as a 
standard MIDI file (SMF), and the mapping between positions in the SMF and the audio signal (audioscore alignment). As a signal-processing front end, we attenuate the DC component and emphasize the high-frequency component by applying a two-tap, high-pass filter with filter coefficients $[1,-1]$. Then our method iterates the following steps for a fixed number of iterations:

1. Update the time-frequency mask to segregate the harmonic components of the violin part, using the audio-score alignment and the estimated fundamental frequency as the cue.

2. Estimate the fundamental frequency of the violin melody, taking into account the pitch notated in the score, the observed spectrogram, and the audio-score alignment.

In the first step, we generate a time-frequency mask, which approaches 1 for time-frequency bins that contain the violin sound and 0 otherwise. We incorporate the musical score information (from the SMF) and the audio-score alignment to find which bins are expected to contain the sound of the violin. Audio-score alignment is determined by extracting a time-sequence of chroma values from both the audio and the SMF, and performing dynamic timewarping between the chroma representation of the audio and SMF to find the optimal path, using the cosine distance as the metric between the audio and the score, similar to the existing work of $\mathrm{Hu}$, Dannenberg, and Tzanetakis (2003).

Audio-score alignment gives the time-sequence of the notated pitch of the violin part, $\hat{f}_{0}(t)$, for all time points $t$. Given this, we simultaneously segregate the violin part and estimate the fundamental frequency of the violin part, $f_{0}(t)$. Our idea, similar to Kameoka's method (Kameoka, Nishimoto, and Sagayama 2007), is to apply a mask that resembles a comb filter to the spectrum centered about $\hat{f}_{0}(t)$ to segregate the violin part. Using the segregated signal, we re-estimate the fundamental frequency. We then re-apply to the original spectrum the mask with the updated fundamental frequency. We iterate these two steps of fundamental frequency update and violin-part segregation until the fundamental frequency converges.
We first segregate the violin part by separating the signal into two sub-signals-the violin part and the residual (i.e., the rest of the signal). We assume that the likelihood of observing the violin part at frequency $f$ is $h_{\mathrm{v}}\left(f \mid f_{0}(t)\right)$, and is of form $\sum_{n=1}^{N} \pi_{n} \mathrm{~N}\left(f \mid n f_{0}(t), \sigma_{f}^{2}\right)$, and we assume that the likelihood of observing the residual is $h_{R}(f)$ and is of form $\frac{1}{\mathrm{~F}}$, where $F$ is the number of frequency bins to consider. $\pi_{n}$ is a multinomial variable that indicates the relative strength of the $n$th partial. $\mathrm{N}\left(x \mid \mu, \sigma^{2}\right)$ is the likelihood of $x$ for a normal distribution with mean $\mu$ and variance $\sigma^{2}$. We assume that the likelihood of observing the violin part is $\alpha$, and the likelihood of observing the residual is $1-\alpha$. For each frequency bin, we associate a latent variable $Z_{\mathrm{V}}$, which indicates whether the violin or the residual contributed to the observed power. $Z_{\mathrm{V}}(f, t)=1$ indicates that at time $t$, the power contained in frequency $f$ originated from the violin part, and $Z_{\mathrm{O}}(f, t, n)=1$ indicates that, of frames generated from the violin, it originated from the $n$th partial of the violin part. The joint likelihood of the observed signal and the latent variable is given as follows:

$$
\begin{aligned}
&\left.\mathrm{p}(X \mid f, t), Z(f, t) \mid f_{0}(t), \alpha, \pi\right) \\
&= \prod_{f, t}\left((1-\alpha) h_{R}(f)\right)^{X(f, t) \mid\left(1-Z_{\mathrm{V}}(f, t)\right)} \\
& \prod_{n=1}^{N}\left(\alpha \pi_{n} \mathrm{~N}\left(f \mid n f_{0}(t), \sigma_{f}^{2}\right)\right)^{X(f, t) Z_{\mathrm{V}}(f, t) Z_{\mathrm{O}}(f, t, n)}
\end{aligned}
$$

We optimize this model using the expectationmaximization (EM) algorithm, in a manner similar to the work of Kameoka, Nishimoto, and Sagayama (2007). In the E-step, we use the parameter from the previous step to find the distribution of $Z$ given $X$ and the parameters. We assign the following:

$$
\begin{aligned}
Q_{0}(f, t, n) & =p\left(Z_{n} \mid X, f_{0}, \alpha, \pi\right) \\
& =\frac{\pi_{n} \mathrm{~N}\left(f \mid n f_{0}(t), \sigma_{f}^{2}\right)}{\sum_{\tilde{n}} \pi_{\tilde{n}} \mathrm{~N}\left(f \mid \tilde{n} f_{0}(t), \sigma_{f}^{2}\right)} \\
Q_{\mathrm{v}}(f, t) & =\frac{\alpha(t) \sum_{n} \pi_{n} \mathrm{~N}\left(f \mid n f_{0}(t), \sigma_{f}^{2}\right)}{(1-\alpha(t)) h_{R}(f)+\alpha(t) \sum_{n} \pi_{n} \mathrm{~N}\left(f \mid n f_{0}(t), \sigma_{f}^{2}\right)}
\end{aligned}
$$


Figure 3. Block diagram of

feature extraction step.

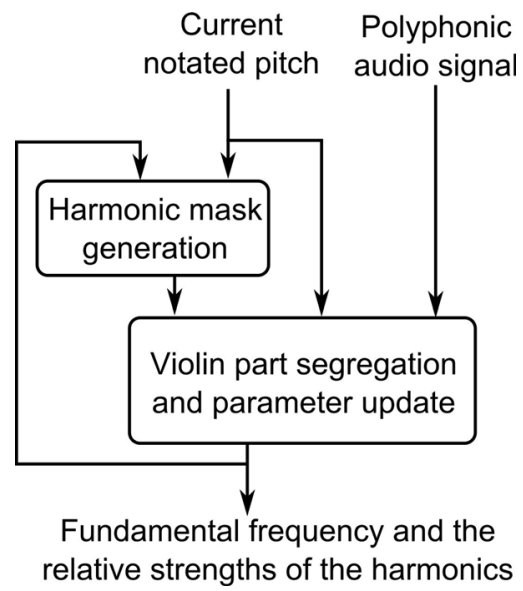

In the M-step, we update the fundamental frequency. We incorporate the knowledge that the fundamental frequency does not deviate too much from the notated pitch, by incorporating a prior distribution of the fundamental frequency as $\mathrm{p}\left(f_{0}(t) \mid \hat{f}_{0}(t)\right)=\mathrm{N}\left(f_{0}(t) \mid \hat{f}_{0}(t), \sigma_{f}^{2}\right)$. Then, the M-step yields the following updates:

$$
\begin{aligned}
f_{0}: & =\frac{\sigma_{f}^{2} \hat{f}_{0}+\sigma_{\hat{f}}^{2} \int_{F} \sum_{n=1} X(f) Q_{\mathrm{V}}(f, t) Q_{\mathrm{O}}(f, t, n) n f \mathrm{~d} f}{\sigma_{f}^{2}+\sigma_{\hat{f}}^{2} \int_{F} \sum_{\tilde{n}=1} X(f) Q_{\mathrm{V}}(f, t) Q_{\mathrm{O}}(f, t, \tilde{n}) \tilde{n}^{2} \mathrm{~d} f} \\
\pi_{n}:= & \frac{\int_{F} X(f) Q_{\mathrm{V}}(f, t) Q_{\mathrm{O}}(f, t, n) \mathrm{d} f}{\sum_{\tilde{n}} \int_{F} X(f) Q_{\mathrm{V}}(f, t) Q_{\mathrm{O}}(f, t, \tilde{n}) \mathrm{d} f} \\
\alpha(t) & =\frac{\int_{F} X(f) Q_{\mathrm{V}}(f, t) \mathrm{d} f}{\int_{F} X(f) \mathrm{d} f}
\end{aligned}
$$

A block diagram of this method is shown in Figure 3.

Finally, we take the logarithm of $\pi(t)$ to enable feature adaptation, as will be discussed. Furthermore, we decorrelate the log-relative strength by taking the discrete cosine transform. That is, $\pi(t):=\mathrm{DCT}(\log \pi(t))$.

\section{Bowed String Identification}

Once we extract the parameters $\Theta(t)=\left\{f_{0}(t), \pi(t)\right\}$, we find the likelihood of the observed data for each of the four bowed strings, i.e., the probability at each
Figure 4. Block diagram of

the GMM. point in time that the violinist is bowing a given string.

The likelihood of bowed string $s_{i}$ is modeled using a Gaussian mixture model (GMM). The GMM models the density of $\pi$ for each pitch pi $\left(f_{0}\right)$, where pi $(f)$ converts frequency $f$ into the closest MIDI note number. Let $\phi_{j}^{(i)}(p), \mu_{j}^{(i)}(p)$, and $\sum_{j}^{(i)}(p)$ indicate respectively the weight, the mean, and the covariance of the $j$ th component of the GMM for the ith bowed string at pitch $p$. Then, the likelihood of observing bowed string $i$ given the feature $\left\{f_{0}(t), \pi(t)\right\}$ and the GMM parameters $\theta_{G M M}=\left\{\phi_{j}^{(i)}(p), \mu_{j}^{(i)}(p), \sigma_{j}^{(i)}(p) \mid \forall i, j, p\right\}$ is given as follows:

$$
\begin{aligned}
& \mathrm{p}\left(s=i \mid \pi(t), p(t), \Theta_{G M M}\left(f_{0}(t)\right)\right) \\
& \quad \propto \sum_{j} \phi_{j}^{(i)}(\operatorname{pi}(t)) \mathrm{N}\left(\pi(t) \mid \mu_{j}^{(i)}(\operatorname{pi}(t)), \Sigma_{j}^{(i)}(\operatorname{pi}(t))\right.
\end{aligned}
$$

$\theta_{G M M}$ is trained using violin audio examples with various playing styles, each of which has a known sequence of pitch and fingerboard position. Using examples with a variety of playing styles has the effect of averaging out statistical discrepancies of the feature arising from playing the example in a particular playing style. Hence, different parameters in GMM correspond to timbral difference arising from the variety of fingerboard position and pitch combinations, and not playing style, e.g., bow pressure or bow velocity. Figure 4 shows the block diagram of the GMM.

The log-likelihood of the bowed string for the $k$ th note, then, is a sum of bowed-string likelihoods for all audio frames that play the kth note. Let $T_{k}(t)$ be a binary variable that is 1 if frame $t$ plays the kth 
Figure 5. Block diagram of the HMM.

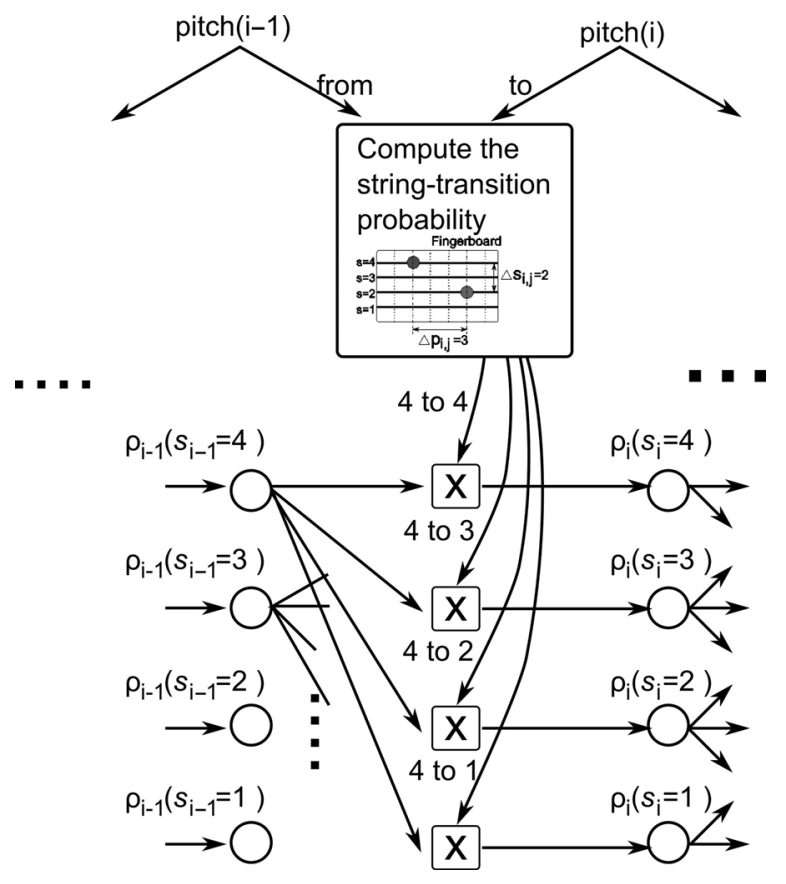

note notated on the score. Then, the bowed string likelihood at note $k, \rho_{k}(s)$, is given as follows:

$$
\rho_{k}(s)=\sum_{t} T_{k}(t) \log \mathrm{p}\left(s \mid \pi(t), p(t), \theta_{G M M}\right)
$$

\section{Sequence Estimation using the Viterbi Algorithm}

We determine the fingerboard-location sequence by finding the most likely bowed-string sequence, taking into account the observed acoustic signal and the difficulty of traversing from one fingerboard location to another. To incorporate both the likelihood of a given bowed string sequence and the likelihood of observing the features given a particular fingerboard position in which a note is played, we model the bowed-string sequence as an HMM. Figure 5 shows a graphical depiction of our model.

We treat the string the note is played on as the hidden state that needs to be estimated, given the observed features $\pi(t)$, the fundamental frequency $f_{0}(t)$, and the inherent difficulty from traversing from one fingerboard position to another. Let $v$ be a parameter that governs the state transition
Figure 6. Simplified

horizontal-vertical model

used in the Viterbi

algorithm.

probability of the HMM. Then, we find the most likely bowed string as follows:

$$
\begin{aligned}
\hat{S}= & \arg \max _{S=\left\{S_{0}, \cdots, S_{N}\right\}} \mathrm{p}\left(S \mid \Theta_{;} \Theta_{G M M}, v\right) \\
= & \arg \max _{S=\left\{S_{0}, \cdots, S_{N}\right\}} \log \rho_{0}\left(s_{0}\right)+\log \mathrm{p}\left(s_{0} ; v\right) \\
& +\sum_{i=1} \log \mathrm{p}\left(s_{i} \mid s_{i-1} ; v\right)+\log \rho_{i}\left(s_{i}\right)
\end{aligned}
$$

$\rho_{i}\left(S_{i}\right)$ is the likelihood of the GMM obtained in the previous section, for the $i$ th note. The maximumlikelihood sequence is determined inductively, using the Viterbi algorithm. Let $S_{\text {opt }}(m, s \mid X, \Theta)$ be the optimal bowed-string sequence for the first $m$ notes that ends in bowed string $s$. Then, we define $S_{\text {opt }}(m+1, s \mid X, \Theta)$ as follows:

$$
\begin{aligned}
S_{\text {opt }}(m+1, s \mid \pi, \Theta)= & \log \mathrm{p}(\pi(m+1) \mid s, \Theta) \\
& +\arg \max _{\hat{s}} \log \mathrm{p}\left(S_{\text {opt }}(m, \hat{s} \mid \pi, \Theta)\right) \\
& +\log \mathrm{p}(s \mid \hat{s}, \Theta)
\end{aligned}
$$

We design a suitable bowed-string transition probability $\mathrm{p}\left(s_{i} \mid s_{j} ; v\right)$ based on a simplified violin fingering model, as shown in Figure 6:

$$
\mathrm{p}\left(S_{i} \mid S_{j} ; v\right) \propto \exp \left(-v\left(\left(\frac{\Delta p_{i, j}}{7}\right)^{2}+\Delta s_{i, j}^{2}\right)\right)
$$

Here, $\Delta p_{i, j}$ is the amount of change between fingerboard positions and $\Delta s_{i, j}$ is the amount of change between string numbers. The constant 7 models the violinists' tendencies to finger an interval of up to 7 semitones by either playing on the same string or crossing a string, but to finger a larger interval by crossing a string. 


\section{Feature Adaptation}

Two violins typically sound different, mainly because each violin has a unique body-resonance characteristic. Therefore, it is essential for us to adapt our model to violins with different bodyresonance characteristics.

Let $Y_{1}$ and $Y_{2}$ be the observed log-magnitude spectrum of two violins. Let $B_{1}$ and $B_{2}$ be the log-magnitude frequency response of the bodies of the two violins, and $S$ be the log-magnitude spectrum of the bow-string interaction. Assuming that the acoustic property of the bow-string interaction does not change significantly across different instruments, we obtain the following relations:

$$
\begin{aligned}
& Y_{1}=B_{1}+S \\
& Y_{2}=B_{2}+S
\end{aligned}
$$

Taking the expectation, we obtain $Y_{2}=$ $Y_{1}-\left\langle Y_{1}\right\rangle_{S}+\left\langle Y_{2}\right\rangle_{S}$, where $\langle Y\rangle_{X}$ denotes expectation of $Y$ under probability distribution of $X$. In our method, we let $Y_{1}$ be the observed features, and generate $Y_{2}$, which is the feature when $Y_{1}$ is played on an instrument with body resonance characteristic $B_{2} .\left\langle Y_{1}\right\rangle_{S}$ is obtained readily by taking the average of the observed signal, and $\left\langle Y_{2}\right\rangle_{S}$ is obtained by summing the average feature of each bowed string, weighted by how often a particular string is played at a given pitch for the given music score. Because our features are linear transformations of the logarithm of the relative powers of harmonic peaks, the same rationale holds. Initially, we set the probability distribution of $S$ as follows:

$$
\begin{aligned}
& \mathrm{p}(S \mid \text { pitch }, \beta) \sim \exp \left(-\frac{\text { pitch }- \text { pitch }_{0}(S)}{\beta}\right) \\
& \text { if pitch }>\text { pitch }_{0}(S), 0 \text { otherwise }
\end{aligned}
$$

Here, pitch is the played pitch, pitch ${ }_{0}$ is the pitch of the open string played on string $S$, and $\beta$ is a positive parameter that assigns a greater probability to lower fingerboard positions, which are more commonly used and somewhat easier for the violinist.

\section{Fingering Estimation}

At the core of our fingering estimation is the violinfingering model, which models the difficulty of a particular fingering. The fingering is determined by designing a cost function between multiple states of hand position, which reflects how difficult it is for the hand to traverse from one position to another.

\section{Violin Fingering Model}

In this study, we extend the existing horizontalvertical cost model presented in the previous section to include fingering practices that are unique to the violin, which we call the violin pedagogical model. It is mainly inspired by practices of violin fingering as suggested by Yampolsky (1967) and Flesch (Flesch 2000). The violin, in particular, is a small, unfretted bowed stringed instrument, making it susceptible to intonation errors. Hence, violin fingerings are often set such that the weak finger (the little finger) is used sporadically, and other fingers in such a way that a natural hand position is maintained.

We define a fingering as a 4-tuple $\boldsymbol{n}=(n, s, f, p)$, where $n \in \mathbb{N}$ is the pitch in MIDI note number, $s \in(1,2,3,4)$ is the bowed string, $f \in(0,1,2,3,4)$ is the pressed finger $(0=$ open string, i.e., no finger placed), and $p \in \mathbb{N}$ is the position. Let $\mathcal{F}$ be a set of all possible fingerings. Also, let $\mathrm{n}(\boldsymbol{n})$ be a function that retrieves the pitch of $\boldsymbol{n} \in \mathcal{F}, \mathrm{s}(\boldsymbol{n})$ the bowed string, $\mathrm{f}(\boldsymbol{n})$ the finger, and $\mathrm{p}(\boldsymbol{n})$ the position.

We define an unnotated score, $S_{u} \in \boldsymbol{n}^{\mathrm{M}}$ to be an $M$-tuple of notes, where $M$ is the number of notes contained in the music score, and the $i$ th element, $S_{u}(i)$, is the $i$ th note of the music score. We define a bowed-string constraint, $c_{\text {bow }} \in(1,2,3,4)^{M}$ associated with an unnotated score $S_{u}$ as an $M$-tuple, where the $i$ th element indicates the bowed string for the ith note. We finally define a notated score, $S_{n} \in \mathcal{F}^{M}$, where the $i$ th element contains the fingering for the $i$ th note.

We then formulate our problem as finding the optimal notated score $S_{\text {opt }}$ that satisfies both the note sequence of the unnotated score obtained using the SMF and the bowed-string constraint 
determined using the Viterbi algorithm. Namely, we define cost functions for traversing a sequence of two notes or three notes, and find the fingering with the smallest net cost that satisfies the constraints. Let $C_{b}\left(s_{i}, s_{i-1}\right): \mathcal{F}^{2} \rightarrow \mathbb{R}$ be a cost function defined over a sequence of two notes. We define a symbol $Z$ that satisfies the following:

$$
\forall A \in \mathcal{F} . C_{b}(Z, A)=C_{b}(A, Z)=0
$$

Then, the optimal fingering is a notated score $F_{\mathrm{opt}}$ that satisfies the following:

$$
\begin{aligned}
\mathrm{F}_{\mathrm{opt}}= & \arg \min _{s_{1} \cdots s_{M} \in \mathcal{F}} \sum_{i=1}^{M} C_{b}\left(s_{i}, s_{i-1}\right) \\
& \text { such that } \forall j<1, \quad s_{j}=0 \text { and } \\
& \forall j \in[1, M] n\left(s_{i}\right)=S_{u}(i) \text { and } s\left(S_{i}\right)=c_{s t}(i)
\end{aligned}
$$

Let $\tau_{\mathrm{p}}=3, \delta_{\mathrm{i}, \mathrm{j}}$ be Kronecker's delta, and $1(\mathrm{c})$ be a function that is 1 if condition $c$ is true and 0 otherwise.

We define the following quantities for convenience:

$$
\begin{aligned}
\Delta_{\mathrm{p}}(\mathrm{i}, \mathrm{j}) & =\mathrm{p}\left(\mathrm{s}_{\mathrm{i}}\right)-\mathrm{p}\left(\mathrm{s}_{\mathrm{j}}\right) \quad \Delta_{\mathrm{s}}(\mathrm{i}, \mathrm{j})=\mathrm{s}\left(\mathrm{s}_{\mathrm{i}}\right)-\mathrm{s}\left(\mathrm{s}_{\mathrm{j}}\right) \\
\Delta_{\mathrm{f}}(\mathrm{i}, \mathrm{j}) & =\mathrm{f}\left(\mathrm{s}_{\mathrm{i}}\right)-\mathrm{f}\left(\mathrm{s}_{\mathrm{j}}\right) \\
\Delta_{\mathrm{pr}}(\mathrm{i}, \mathrm{j}) & =\left|\mathrm{n}\left(\mathrm{s}_{\mathrm{i}}\right)-\mathrm{p}_{0}\left(\mathrm{st}\left(\mathrm{s}_{\mathrm{j}}\right) \mid\right)-\right| \mathrm{n}\left(\mathrm{s}_{\mathrm{i}}\right)-\mathrm{p}_{0}\left(\mathrm{~s}_{\mathrm{j}}\left(\mathrm{s}_{\mathrm{j}}\right)\right) \mid \\
\mathrm{R}(\mathrm{i}, \mathrm{j}) & =\left[\mathrm{P}_{\min }\left(\mathrm{f}\left(\mathrm{s}_{\mathrm{i}}\right), \mathrm{P}_{\max }\left(\mathrm{f}\left(\mathrm{s}_{\mathrm{j}}\right)\right)\right]\right.
\end{aligned}
$$$$
\begin{aligned}
P_{\max } & =\left(\begin{array}{lllll}
0 & 0 & 0 & 0 & 0 \\
0 & 0 & 2 & 4 & 6 \\
0 & 2 & 0 & 2 & 5 \\
0 & 4 & 2 & 0 & 3 \\
0 & 6 & 5 & 3 & 0
\end{array}\right) \\
P_{\text {nat }} & =\left(\begin{array}{lllll}
0 & 0 & 0 & 0 & 0 \\
0 & 0 & 2 & 3 & 5 \\
0 & 2 & 0 & 1 & 2 \\
0 & 3 & 1 & 0 & 2 \\
0 & 5 & 2 & 2 & 0
\end{array}\right)
\end{aligned}
$$

$$
\mathrm{P}_{\min }=\left(\begin{array}{ccccc}
0 & 0 & 0 & 0 & 0 \\
0 & 0 & 1 & 2.1 & 3.5 \\
0 & 1 & 0 & 1.1 & 2.5 \\
0 & 2.1 & 1.1 & 0 & 1.5 \\
0 & 3.5 & 2.5 & 1.5 & 0
\end{array}\right)
$$

Then, we define a two-note generic fingering model (denoted HVM-2) as the following set of features $\mathrm{X}_{\mathrm{BN}}$ :

$$
\begin{aligned}
\mathrm{b}_{0}= & \left|\Delta_{\mathrm{p}}(\mathrm{i}, \mathrm{i}-1)\right| \cdot 1\left(\mathrm{f}\left(\mathrm{s}_{\mathrm{t}}\right) \neq 0 \wedge \mathrm{f}\left(\mathrm{s}_{\mathrm{i}-1}\right) \neq 0\right) \\
\mathrm{b}_{1}= & 1\left(\mathrm{fg}\left(\mathrm{s}_{\mathrm{i}}\right) \neq 0 \wedge \Delta_{\mathrm{f}}(\mathrm{i}, \mathrm{i}-1)\right. \\
= & \left.0 \wedge \Delta_{\mathrm{p}}(\mathrm{i}, \mathrm{i}-1) \neq 0\right) \\
\mathrm{b}_{2}= & 1\left(\Delta_{\mathrm{f}}(\mathrm{i}, \mathrm{i}-1) \cdot \Delta_{\mathrm{pr}}(\mathrm{i}, \mathrm{i}-1)<0 \wedge\left|\Delta_{\mathrm{p}}\right|<\tau_{\mathrm{p}}\right. \\
\mathrm{b}_{3}= & \left|\Delta_{\mathrm{pr}}(\mathrm{i}, \mathrm{i}-1)\right|-\mathrm{P}_{\mathrm{nat}}\left(\mathrm{f}\left(\mathrm{s}_{\mathrm{i}}\right), \mathrm{f}\left(\mathrm{s}_{\mathrm{i}-1}\right)\right) \\
& \cdot 1\left(\left|\Delta_{\mathrm{pr}}(\mathrm{i}, \mathrm{i}-1)\right| \in \mathrm{R}(\mathrm{i}, \mathrm{i}-1)\right) \\
\mathrm{x}_{\mathrm{BN}}(1)= & \infty \cdot 1\left(\mathrm{n}\left(\mathrm{s}_{\mathrm{i}}\right)-\mathrm{p}_{0}\left(\mathrm{st}\left(\mathrm{s}_{\mathrm{i}}\right)\right)-\operatorname{pos}\left(\mathrm{s}_{\mathrm{i}}\right) \notin \mathrm{R}(1, \mathrm{i})\right) \\
\mathrm{x}_{\mathrm{BN}}(2,3)= & \left(\mathrm{b}_{0}, \mathrm{~b}_{0}^{2}\right) \\
\mathrm{x}_{\mathrm{BN}}(4)= & \mathrm{b}_{1} \\
\mathrm{x}_{\mathrm{BN}}(5,6)= & \left(\left|\Delta_{\mathrm{s}}(\mathrm{i}, \mathrm{i}-1)\right|,\left|\Delta_{\mathrm{s}}(\mathrm{i}, \mathrm{i}-1)\right|^{2}\right) \\
& \cdot 1\left(\mathrm{fg}\left(\mathrm{s}_{\mathrm{i}}\right) \cdot \mathrm{fg}\left(\mathrm{s}_{\mathrm{i}-1}\right) \neq 0\right) \\
\mathrm{x}_{\mathrm{BN}}(7)= & 1\left(\left|\Delta_{\mathrm{pr}}(\mathrm{i}, \mathrm{i}-1)\right|-\mathrm{P}_{\mathrm{nat}}\left(\mathrm{fg}\left(\mathrm{s}_{\mathrm{i}}\right), \mathrm{fg}\left(\mathrm{s}_{\mathrm{i}-1}\right)\right) \neq 0\right) \\
\mathrm{x}_{\mathrm{BN}}(8)= & \mathrm{b}_{2} \cdot 1(\mathrm{fg}(\mathrm{i}) \neq 0 \wedge \mathrm{fg}(\mathrm{i}-1) \neq 0) \\
\mathrm{x}_{\mathrm{BN}}(9)= & \mathrm{b}_{3} \cdot 1(\mathrm{fg}(\mathrm{i})=0 \vee \mathrm{fg}(\mathrm{i}-1)=0)
\end{aligned}
$$

$\mathrm{x}_{\mathrm{BN}}(1)$ determines whether a note is physically playable on a particular string, where $\infty \times 0=0$; i.e., this feature discards any fingering that is physically unplayable, as shown in Figure $7 . \mathrm{x}_{\mathrm{BN}}(2,3)$ applies a penalty for a change of position. $\mathrm{x}_{\mathrm{BN}}(4)$ penalizes a change of position using the same finger (i.e., a glissando). $\mathrm{x}_{\mathrm{BN}}(5,6)$ adds a penalty for a change of fingerboard location. $\mathrm{x}_{\mathrm{BN}}(7)$ penalizes playing in an unnatural hand position. $\mathrm{x}_{\mathrm{BN}}(8)$ penalizes playing a sequence in which the second note is placed higher on the fingerboard, but the finger traverses from high finger to low (e.g., little finger to the index finger), and vice versa. Finally, $\mathrm{x}_{\mathrm{BN}}(9)$ prevents change of 
Figure 7. Graphical description of $X_{B N}(1)$. A Roman numeral indicates the string $(I V=$ lowest, $I=$ highest), and a number indicates the pressed finger $(1=$ index finger, $4=$ little finger)

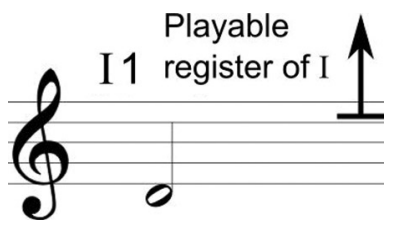

Figure 7

\section{Figure 8}

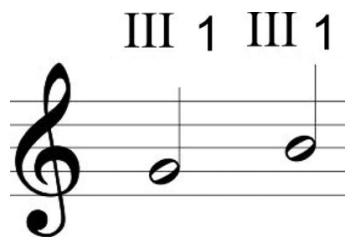

position (wrist movement), when it is completely natural not to do so.

We define a two-note fingering model inspired by the literature of violin pedagogy (denoted VPM-2) as the following set of features $\mathrm{x}_{\mathrm{BV}}$ :

$$
\begin{aligned}
& \operatorname{cond}_{0}=\mathrm{fg}\left(\mathrm{s}_{\mathrm{i}}\right) \cdot \mathrm{fg}\left(\mathrm{s}_{\mathrm{i}-1}\right) \neq 0 \wedge\left|\Delta_{\mathrm{p}}(\mathrm{i}, \mathrm{i}-1)\right|<\tau_{\mathrm{p}} \\
& \mathrm{x}_{\mathrm{BV}}(1)=1\left(\operatorname{cond}_{0} \wedge \Delta_{\mathrm{pr}}(\mathrm{i}, \mathrm{i}-1)\right. \\
& \left.\neq 0 \wedge \Delta_{\mathrm{f}}(\mathrm{i}, \mathrm{i}-1)=0\right) \\
& \mathrm{x}_{\mathrm{BV}}(2)=1\left|\operatorname{cond}_{0} \wedge\right| \Delta_{\mathrm{pr}}(\mathrm{i}, \mathrm{i}-1) \mid \\
& \left.=1 \wedge\left|\Delta_{\mathrm{f}}(\mathrm{i}, \mathrm{i}-1)\right|>1\right) \\
& \mathrm{X}_{\mathrm{BV}}(3)=1\left(\mathrm{fg}\left(\mathrm{s}_{\mathrm{i}}\right) \cdot \mathrm{fg}\left(\mathrm{s}_{\mathrm{i}-1}\right) \neq 0 \wedge \Delta_{\mathrm{p}}(\mathrm{i}, \mathrm{i}-1)\right. \\
& \left.\neq 0 \wedge \Delta_{\mathrm{pr}}(\mathrm{i}, \mathrm{i}-1) \cdot \Delta_{\mathrm{f}}(\mathrm{i}, \mathrm{i}-1)=-1\right) \\
& \mathrm{x}_{\mathrm{BV}}(4)=1\left(\mathrm{fg}\left(\mathrm{s}_{\mathrm{i}}\right)=\mathrm{fg}\left(\mathrm{s}_{\mathrm{i}-1}\right)\right. \\
& =4 \wedge|\operatorname{note}(\mathrm{i})-\operatorname{note}(\mathrm{i}-1)|>1) \\
& \mathrm{X}_{\mathrm{BV}}(5)=\operatorname{pos}\left(\mathrm{s}_{\mathrm{i}}\right)
\end{aligned}
$$

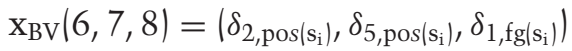

$\mathrm{x}_{\mathrm{BV}}(1)$ penalizes using the same finger to move to a different position, as shown in Figure $8 . \mathrm{x}_{\mathrm{BV}}(2)$ penalizes a chromatic change of the relative position using a nonadjacent finger, as shown in Figure 9; such movement involves wrist motion, which is considerably harder to tune than placing a finger with a slight stretch. $\mathrm{x}_{\mathrm{BV}}(3)$ lessens the penalty for shifting up from a higher-numbered finger to a
Figure 9. Graphical

description of $X_{B V}(2)$.
Figure 10. Graphical

description of $X_{B V}(3)$.

Figure 9
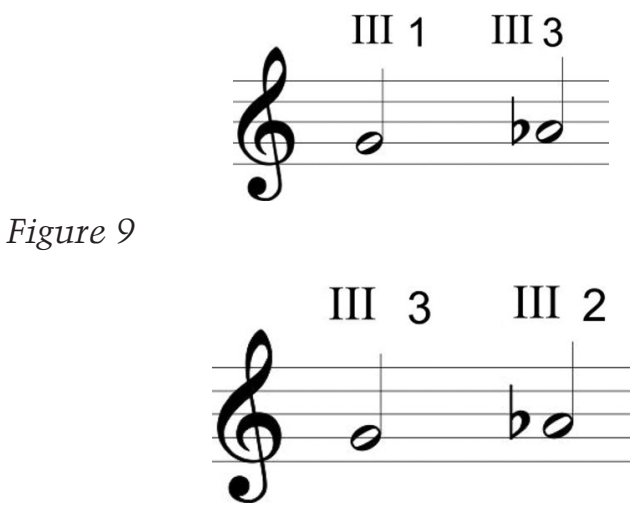

Figure 10

lower-numbered finger, or vice versa, when the shift is small, as shown in Figure 10. $\mathrm{x}_{\mathrm{BV}}(4)$ penalizes an adjacent use of the little finger, which is weak and prone to intonation errors. $\mathrm{x}_{\mathrm{BV}}(5)$ adds a penalty for an extremely high position, which is harder to play in tune. $\mathrm{x}_{\mathrm{BV}}(6,7)$ adds a preference for playing in the first, second, or third positions, which are typically the three easiest positions to play in. $\mathrm{x}_{\mathrm{BV}}(8)$ penalizes the half-position, the lowest possible position but an unconventional one; hence, it is unnatural from the perspective of violin pedagogy but perhaps the easiest position to play in tune from a physical perspective.

Using these features, we define the two-note cost function as follows:

$$
\mathrm{C}_{\mathrm{b}}=\operatorname{diag}\left(\mathbf{W}_{\mathbf{B N}} \quad \mathbf{W}_{\mathbf{B V}}\right)\left[\begin{array}{ll}
\mathbf{x}_{\mathbf{B N}} & \mathbf{x}_{\mathbf{B V}}
\end{array}\right]^{\mathrm{T}}
$$

$\mathbf{W}_{\mathbf{B N}}$ and $\mathbf{W}_{\mathbf{B V}}$ define the relative weight of each feature. Given ample training data, statistical machine learning of these weights might be possible. In this article, we choose to manually adjust the weights, as the violin fingering corpus is too small to permit statistical machine learning.

\section{Experiments}

We perform three experiments. The first experiment assesses the performance of feature-adaptation and error-correction algorithms on bowed-string estimation, the second experiment assesses the performance of the bowed-string classifier in a 
Figure 11. Recognition accuracy (\%) for different values of $v$ (all values are for data with data adaptation). $S S=$ same violin and same strings as

training data; $S D=$ same

violin with different

strings; $D D=$ different

violin and different strings.

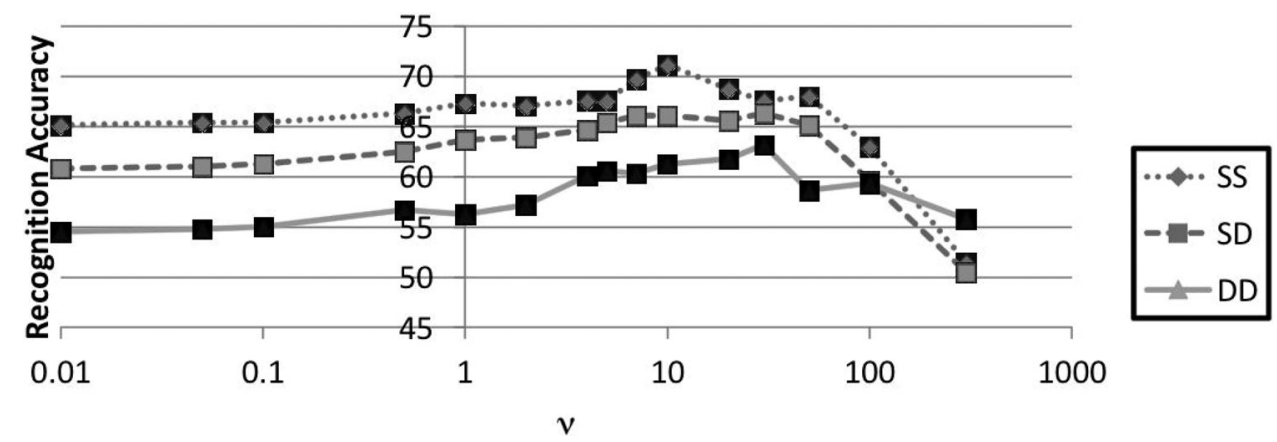

polyphonic mixture, and the third experiment evaluates the playability of our new fingering model through subjective experiments. In the first two experiments, we prepared recordings of three pieces of classical music, using two significantly different fingerings (207 notes for three pieces, yielding a total of 414 notes). For each fingering, we recorded the music with the following three conditions:

1. Using the same violin and strings as were used to record the training data (denoted as setup SS).

2. Using the same violin but with a different brand of strings (setup SD).

3. Using a different violin with a different brand of strings (setup DD).

This results in about 18 minutes of audio for validation. The training data, which lasts about 24 minutes, consists of two-octave chromatic scales played on each string of an electric violin, using various dynamics.

Setup SS and SD were played on the same electric violin, and DD on an acoustic violin. We chose to record the training data using an electric violin and to use an acoustic violin for DD for two reasons. First, it is easy to record noise-free training data using an electric violin. Second, because electric and acoustic violins sound extremely different, the evaluation of the feature adaptation mechanism can be regarded as the worst-case performance. Therefore, in a real-life application, we expect the system to perform somewhere between setup SD and $\mathrm{DD}$, as long as the training data use an acoustic violin. In the EM algorithm, we set $\sigma_{f}^{2}=0.1$ and $\sigma_{\hat{f}}^{2}$ to start at 50 and narrow down inverse-linearly with each iteration of the algorithm. The value $\beta$ used in model adaption is chosen to be 0.1 .

\section{Experiment 1: Evaluation of Bowed-String Identifier}

We evaluated the accuracy of the bowed-string estimator with and without feature adaptation, each time evaluating the accuracy (1) of a baseline, i.e., without considering any sequential information; (2) considering sequential information using a previous study (Maezawa et al. 2009, 2010); and (3) considering sequential information using the Viterbi algorithm as proposed in this article. The value of $v$ used for the Viterbi algorithm in the bowed sequence estimation was set to 30 , chosen by evaluating the accuracy for various values of $v$, as shown in Figure 11 . Figure 12 shows the result. We find that our method consistently outperforms our previous study. In all cases, adaptation decreases the performance when the training data and the validation data are from the same instrument and the same brand of strings. This is because adaptation itself depends on the estimated sequence of fingerboard positions, which contains errors; the discrepancy between the actual sequence of fingerboard positions and the estimated ones is small enough to be effective in absorbing the differences in body resonance characteristics between two different violins or strings, but significant when the same string and violin is used. 
Figure 12. Comparison of recognition accuracy (\%) for different sequence estimation methods. $S S=$ same violin and same strings as training data; $S D=$ same violin with different strings; $D D=$ different violin and different strings.
Figure 13. Recognition accuracy with different levels of accompaniment. $S S=$ same violin and same strings as training data;
$S D=$ same violin with different strings;

$D D=$ different violin and different strings.

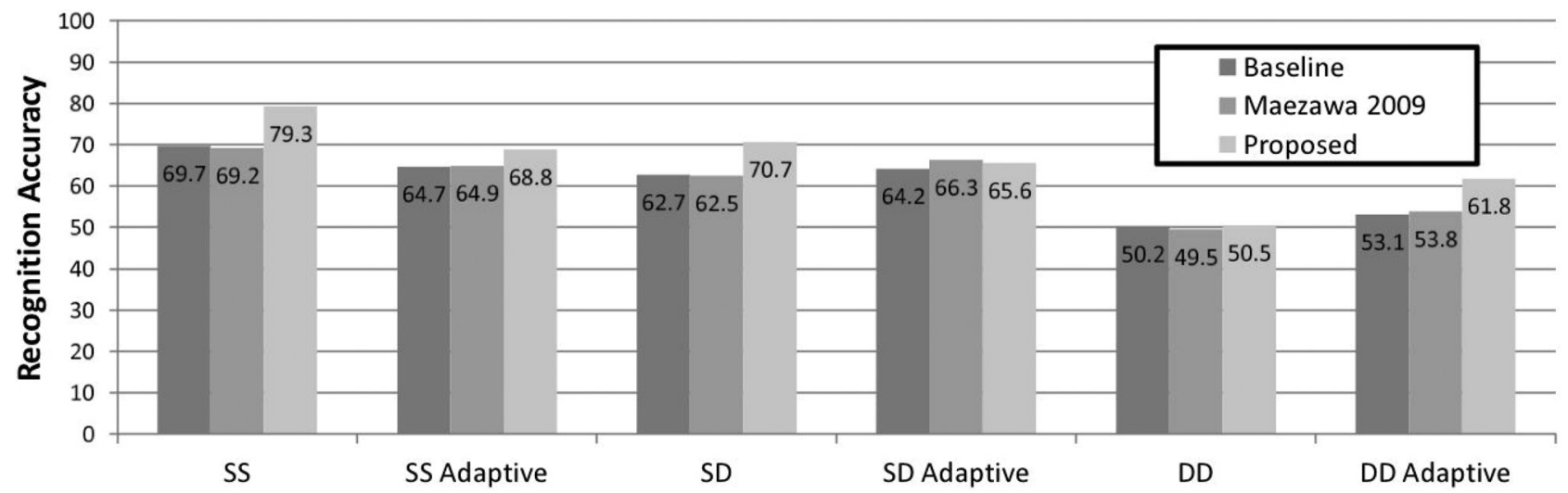

Figure 12

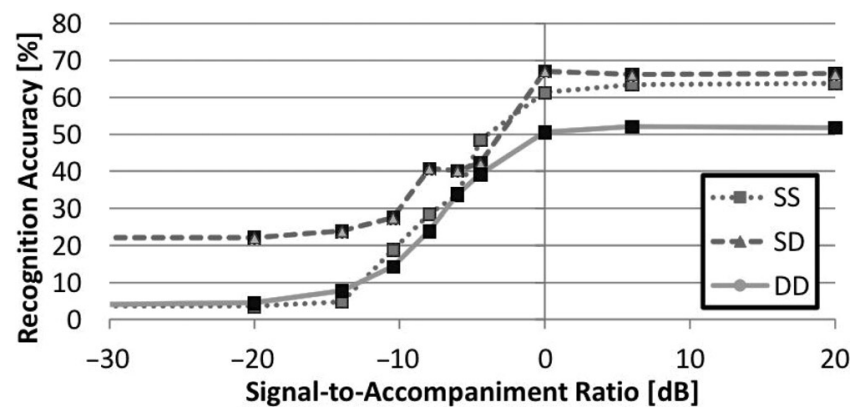

Figure 13

\section{Experiment 2: Effect of Accompaniment on Feature Extraction}

Piano accompaniments were generated for two of the three pieces, using a synthesizer with uniform note velocity, and the amplitude was adjusted such that the peak values of the solo violin and the accompaniment were identical. Next, the recognition accuracy was evaluated for each violin/string type, by changing the level of the accompaniment. We scaled the amplitude of the violin part such that the root-mean-square power of the violin part relative to that of the accompaniment is set to a given value of signal-to-accompaniment ratio. Figure 13 shows the result. We find that the bowed-string estimator performs similarly for different ratios, as long as the ratio is positive, that is, the signal level of the violin is greater than the "noise" level (i.e., the accompaniment).

\section{Experiment 3: Subjective Evaluation of the Fingering Estimation}

First, ten excerpts from three classical pieces were prepared (approximately 200 notes total). For each excerpt, we generated two fingerings: one using HVM-2, and the other using HVM-2+VPM-2. We assume that bowed-string estimation is perfectly accurate, by not incorporating constraints based on the estimated bowed string. The feature weight $\boldsymbol{W}$ was manually tuned, by first adjusting the parameters for example (1) until it generated satisfactory fingerings for the repertoires considered. Then, parameters pertaining to example (2) were adjusted. They were set to $\boldsymbol{W}_{\mathbf{B N}}=[1,0.5$, $1,1,5,1,0.5,20,10]$ and $\boldsymbol{W}_{\mathbf{B V}}=[1,10,-0.5,10$, $1.1,-0.3,-0.2,0.2]$. Excerpts that generated different fingerings for each of the setups were then extracted.

Next, seven violinists of various skills (amateur and professional, i.e., ten or more years of experience) evaluated the generated fingerings using a form as shown in Figure 14. Each violinist was presented with fingerings generated using VPM-2 and VPM-2 + HVM-2, and was asked to choose the better of the two, if any. Seven violinists were each given ten questions (70 total), but only 66 answers were 
Figure 14. A screen capture

of the questionnaire.

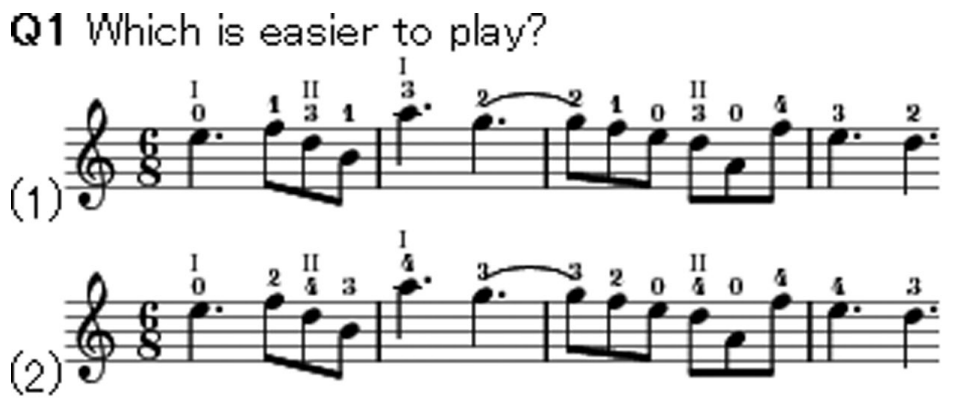

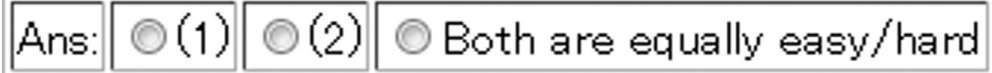

If you have answered (1) to the question, please proceed to Question Q1-a. Otherwise, please proceed to Question $\underline{\mathbf{Q} 1-\mathbf{b}}$.

Table 1. The Number of Answers Indicating a Preference for the Baseline Model (HVM-2) or for the Violin Pedagogical Model (HVM-2 + VPM-2)

\begin{tabular}{lc}
\hline Setup & Total Count \\
\hline HVM-2 & 5 \\
HVM-2 + VPM-2 & 54 \\
No preference & 7 \\
\hline
\end{tabular}

provided. Table 1 shows the result of the survey. The sign test shows that HVM-2 and VPM-2+HVM-2 are not equally favored $(p=0.01)$, which suggests that our proposed model (HVM-2+VPM-2) is favored over the baseline (HVM-2 only).

\section{Discussion}

From the results in Experiment 1 we observe that in setup DD - the most realistic situation-adaptation improves the recognition accuracy, whereas in SS and SD, the accuracy decreases. We believe this occurs because of the mismatch between the distribution of the actual bowed-string sequence and that assumed in our model. Moreover, we find that our study offers major improvements compared with our previous study. In all cases, our method improves the recognition accuracy over the baseline, which suggests that considering the playability of a particular sequence of notes over a particular sequence of bowed strings is effective.

Experiment 2 suggests that our features are robust in polyphonic audio mixtures, as long as the signal level of the violin solo part is as loud as the accompaniment. This condition seems to hold in pieces where the violin has an important melody (and hence, the choice of fingerboard location becomes an even greater musical issue). Therefore, we believe our method performs without significant degradation of accuracy in practical applications involving works for violin and piano.

Finally, we found that the fingering generated is more natural when the proposed fingering model is used than when existing ones are. We believe incorporating the preference for the first and third positions (index finger placed 2 semitones and 5 semitones above the nut of the violin, respectively) is the chief reason-they are the most frequently used positions on the violin, and fingerings generated on these positions are more natural for violinists to play. We find that incorporating these kinds of heuristics could drastically improve playability. 
Figure 15. Estimated

fingering from first 50 bars

of Romanze in C played by

Joachim (1903).
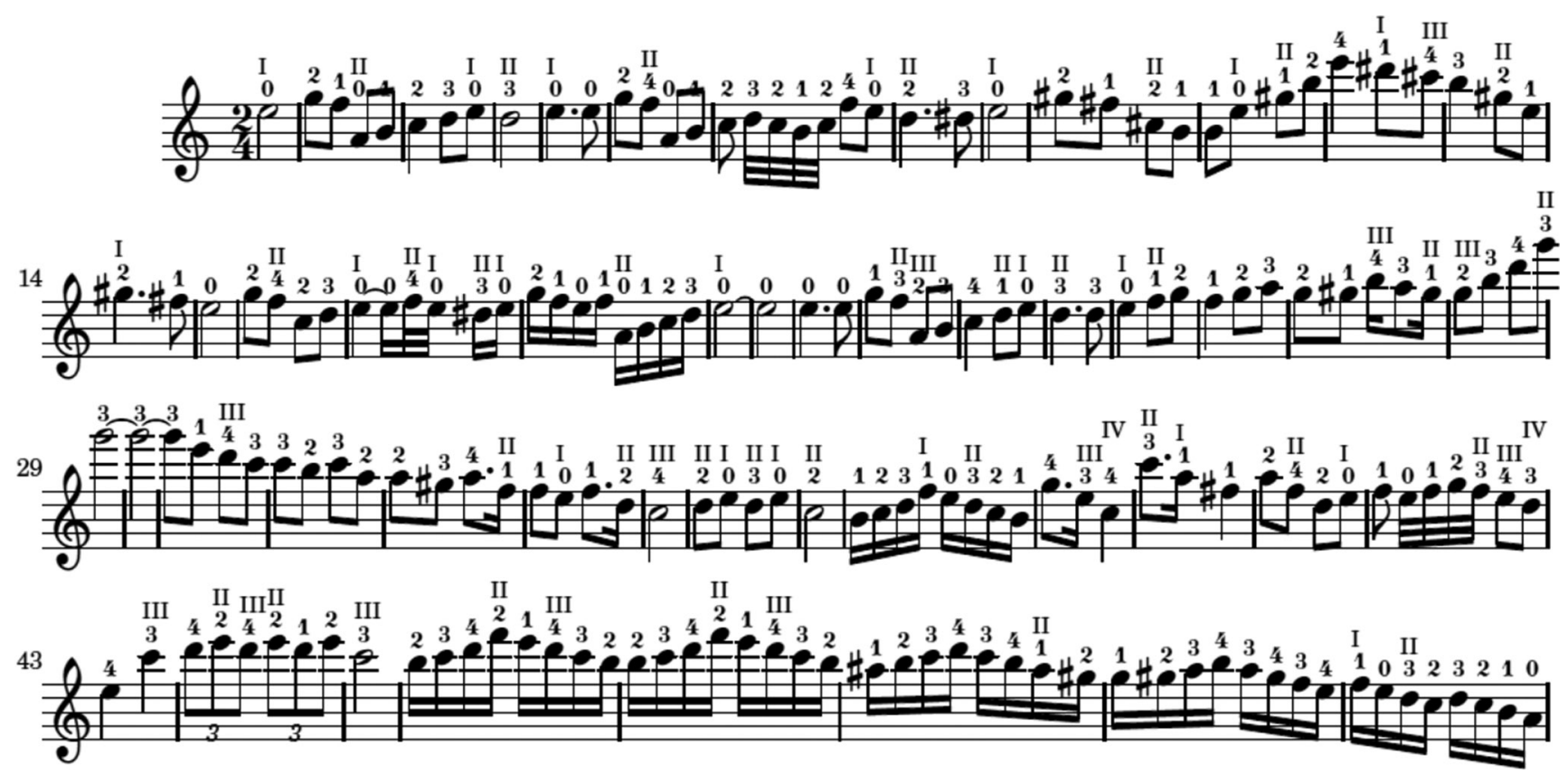

Next, we shall discuss an application of our system for recovering the fingering of a recording from more than a century ago.

\section{Application: Demystifying Historical Recordings}

Our method may be used to analyze a recording from the past to recover the fingering of a legendary violinist. In this example, we attempt to recover the fingering of Romanze in $C$ composed by Joachim, a legendary violinist of the late 19th century, using a gramophone recording of Joachim in 1903. The recording was pitch-shifted such that the notated A4 was set to $440 \mathrm{~Hz}$. VPM-2+HVM-2 fingering model was used to estimate the fingering, an excerpt of which is shown in Figure 15.

We speculate, however, that the actual fingering may have been very different from that estimated by our method. For example, there are notes that are playable using open strings (i.e., no finger pressed) whose estimated fingerings on the notated score show open strings; yet, on the recording, we clearly hear the note played with a vibrato, meaning that it had to have been played by stopping a string with a finger. These kinds of errors might have occurred for three reasons:

A) In Joachim's time, the material used for the violin string was significantly different. In particular, the E-string (the highest-pitched string) used gut as its core, which has a much mellower timbre than the kind of string used today. Such discrepancies in the material property of the string itself would cause the performance of the bowed-string estimator to deteriorate.

B) A high-quality recording of Joachim is not available. The remastered recording we used had a frequency range that extended up to only approximately $5 \mathrm{kHz}$; the rest was cut off, perhaps by applying an equalizer. Hence, there were only few partials that conveyed meaningful information.

C) Joachim's use of pitch-based playing techniques, such as vibrato and glissando, gives many clues to violinists who want to infer Joachim's playing. For example, if a transition 
from one note to another is completely smooth, it strongly suggests that the two notes were played on the same string. Vibrato can be a giveaway for distinguishing whether a note is played using an open string or not. Our method, however, does not incorporate the pitch trajectory for fingerboard-location estimation, and hence, misses such clues.

This output suggests a future direction for research: incorporating pitch-based cues for fingerboard inference, and perhaps robustness to different materials used for a given string. The latter, however, can be ameliorated with our method to a certain extent; we can record the training data using the string that is thought to be used in the recording whose fingering we would like to infer.

\section{On Inharmonicity}

Stringed instruments such as the violin do not produce overtones that are exact integer multiples of the fundamental frequency. Such deviation from integer harmonics is caused by the inharmonicity of the violin string, which in turn is created by torsion of the string. Because inharmonicity is dependent on the material of the string, our model initially incorporated inharmonicity, using a beta distribution with a small shape parameter as its prior distribution. We found, however, that such a model produced lower accuracy than that without inharmonicity. Because of the nature of the EM algorithm, we found that the model tends to "explain" partials of the accompaniment as arising from the violin sound with extremely high inharmonicity.

\section{Conclusion}

This article presented a method for recovering the violin fingering from an input audio signal and a music score by analyzing the bowed-string sequence, and using it as a constraint to determine the optimal fingering. Bowed-string sequence classification was based on features that are robust in a polyphonic mixture. We also incorporated a sequential model based on violin playing. We found that such sequential modeling drastically improved the accuracy. The fingering estimation incorporated features that are specific to violin playing practices (the pedagogical model), in addition to some of the more fundamental features applicable to other instruments as well. Incorporating such heuristics generated a drastically easier fingering.

Future research directions may involve improved recognition accuracy, refining the fingering model, and more applications. For example, recognition accuracy may be improved by exploiting the smoothness of pitch trajectory (glissando, vibrato, etc.), as we observed that violinists listen to the smoothness of pitch transition to infer the fingerboard location and the fingering. The fingering model may further be improved by incorporating more features that are inspired from violin pedagogy or through machine learning of feature weights by preparing a large violin fingering corpus. Another possibility is to perform joint estimation of fingerboard location and fingering - the problems of fingerboard location estimation and fingering estimation are dependent on each other, suggesting that joint estimation may improve the performance of both. From a musicological perspective, artist classification, skill assessment, and analysis of historical recordings may be interesting applications of our approach to fingering estimation.

\section{Acknowledgments}

This work is supported by Grant-in-aid for Scientific Research (S) and CREST-MUSE of JST.

We would like to thank violinists P. Klinger, Dr. P. Sunwoo, and Dr. J. Choi for stimulating and inspiring discussions on violin fingering.

\section{References}

Barbancho, I. 2009. "Transcription and Expressiveness Detection System for Violin Music." In Proceedings of the International Conference on Acoustics, Speech and Signal Processing, pp. 189-192. 
Burns, A. M., and M. M. Wanderley. 2006. "Visual Methods for Retrieval of Guitarist Fingering." In Proceedings of the International Conference on New Interface for Musical Expression, pp. 196-199.

Cremer, L., and J. S. Allen. 1984. The Physics of the Violin. Cambridge, Massachusetts: MIT Press.

Flesch, C. 2000. The Art of Violin Playing. 2nd ed., vol. I. New York: Carl Fischer.

Fletcher, N. H., and T. D. Rossing. 1998. The Physics of Musical Instruments. 2nd ed. New York: Springer.

Hu, N., R. B. Dannenberg, and G. Tzanetakis. 2003. "Polyphonic Audio Matching and Alignment for Music Retrieval." In Proceedings of the Institute of Electrical and Electronics Engineers Workshop on Applications of Signal Processing to Audio and Acoustics, pp. 185188.

Kameoka, H., T. Nishimoto, and S. Sagayama. 2007. "A Multipitch Analyzer Based on Harmonic Temporal Structured Clustering." Institute of Electrical and Electronics Engineers Transactions on Audio, Speech and Language Processing 15(3):982-994.

Kasimi, A. A., E. Nichols, and C. Raphael. 2007. "A Simple Algorithm for Automatic Generation of Polyphonic Piano Fingerings." In Proceedings of the International Society for Music Information Retrieval Conference, pp. 355-356.

Krishnaswamy, A., and J. Smith. 2003. "Inferring Control Inputs to an Acoustic Violin from Audio Spectra." In Proceedings of the Institute of Electrical and Electronics Engineers International Conference on Multimedia and Expo, pp. 733-736.

Lu, H., et al. 2008. "iDVT: An Interactive Digital Violin Tutoring System Based on Audio-Visual Fusion." In Austria Association for Computing Machinery International Conference on Multimedia, pp. 300301.
Maezawa, A., et al. 2009. "Bowed String Sequence Estimation of a Violin Based on Adaptive Audio Signal Classification and Context-Dependent Error Correction." In Proceedings of the International Symposium on Multimedia, pp. 9-16.

Maezawa, A., et al. 2010. "Violin Fingering Estimation Based on Violin Pedagogical Fingering Model Constrained by Bowed Sequence Estimation from Audio Input." Trends in Applied Intelligent Systems, 3:249-259.

Radicioni, D. P., L. Anselma, and V. Lombardo. 2004. "A Segmentation-Based Prototype to Compute String Instruments Fingering." In Proceedings of the Conference on Interdisciplinary Musicology. Available online at www.uni-graz.at/richard.parncutt/cim04. Accessed May 2012.

Radisavljevic, A., and P. F. Driessen. 2004. "Path Difference Learning for Guitar Fingering Problems." In Proceedings of the International Computer Music Conference, pp. 730-733.

Sayegh, S. I. 1989. "Fingering for String Instruments with the Optimal Path Paradigm." Computer Music Journal 13(3):76-83.

Traube, C., and J. Smith. 2000. "Estimating the Plucking Point on a Guitar String." In Proceedings of the International Conference on Digital Audio Effects, pp. 153-158.

Yampolsky, I.M. 1967. Principles of Violin Fingering. New York: Oxford University Press.

Yonebayashi, Y., H. Kameoka, and S. Sagayama. 2007. "Automatic Decision of Piano Fingering Based on a Hidden Markov Model." In Proceedings of the International Joint Conference on Artificial Intelligence, pp. 2915-2921.

Zhang, B., J. Zhu, and W. Leow. 2007. "Visual Analysis of Fingering for Pedagogical Violin Transcription." In Proceedings of the ACM Conference on Multimedia, pp. 521-524. 